\title{
Early experiences of endovascular aneurysm repair for ruptured abdominal aortic aneurysms
}

\author{
Dayoung Ko ${ }^{1, *}$, Hyung Sub Park ${ }^{1, *}$, Jang Yong Kim², Daehwan Kim", Taeseung Lee ${ }^{1}$ \\ ${ }^{1}$ Department of Surgery, Seoul National University Bundang Hospital, Seoul National University College of Medicine, Seongnam, \\ Korea \\ 2Department of Surgery, The Catholic University of Korea School of Medicine, Seoul, Korea
}

\begin{abstract}
Purpose: The use of endovascular aneurysm repair (EVAR) for ruptured abdominal aortic aneurysms (r-AAA) is steadily increasing. We report early experiences of EVAR for r-AAA performed in two tertiary referral centers in Korea.

Methods: We retrospectively reviewed r-AAA patients treated by EVAR from May 2013 to December 2017. An EVAR-first strategy for r-AAA was adopted whenever feasible. The demographic information, anatomic characteristics, operative details, postoperative complications with special attention to abdominal compartment syndrome (ACS), and 30-day mortality were collected and analyzed.

Results: We identified 13 patients who underwent EVAR for r-AAA. Mean age was 74.2 years and mean AAA size was 74.2 $\mathrm{mm}$. Two patients underwent cardiopulmonary resuscitation at initial presentation. Bifurcated stent grafts were used in 12 out of 13 cases and physician-modified endografts with fenestrated/chimney techniques were performed in 2 cases with short neck. Successful stent graft deployment was achieved in all cases. Three patients were suspected of having ACS and 2 of them underwent laparotomy for decompression. The 30-day mortality was $7.7 \%$ (1 of 13), the only mortality being a patient that refused decompressive laparotomy for suspected ACS.

Conclusion: Despite the small numbers, the outcomes of EVAR for treatment of r-AAA were very promising, even in selected cases with unfavorable anatomy. These outcomes were achieved by a dedicated and well-trained team approach, and by use of high-end angiographic technology. Finally, ACS after EVAR is not uncommon, and requires a high index of suspicion as well as liberal use of decompressive surgery.

[Ann Surg Treat Res 2019;96(3):138-145]
\end{abstract}

Key Words: Abdominal aortic aneurysm, Endovascular procedures, Rupture

\section{INTRODUCTION}

Abdominal aortic aneurysms (AAA) have the potential to rupture, with the size of an aneurysm being the single most important factor in determining the risk of rupture [1]. The mortality rate of ruptured AAA (r-AAA) is in the range of $80 \%-90 \%$, with only half of patients being able to make it to the hospital. Most of these patients have abnormal vital signs at presentation in the form of hemorrhagic shock. Thus, only half of the patients survive the repair of r-AAA [2].

The treatment of choice for r-AAA has traditionally been considered open surgical repair. However, with the increasing use of EVAR for elective AAA cases, the use of EVAR for r-AAA has also increased. Many reports in the literature have shown the feasibility of EVAR for r-AAA, but many of these were single center studies, and randomized control trials (RCT) are scarce due to the difficulty of performing clinical studies in emergency settings. The currently available RCTs include the AJAX [3],
Received May 25, 2018, Revised September 25, 2018,

Accepted October 16, 2018

Corresponding Author: Taeseung Lee

Department of Surgery, Seoul National University Bundang Hospital, 82

Gumi-ro 173 beon-gil, Bundang-gu, Seongnam 13620, Korea

Tel: +82-31-787-7092, Fax: +82-31-787-4078

E-mail: tslee@snubh.org

ORCID code: https://orcid.org/0000-0001-6425-5924
*Dayoung Ko and Hyung Sub Park contributed equally to this work as cofirst authors.

Copyright (c) 2019, the Korean Surgical Society

(c) Annals of Surgical Treatment and Research is an Open Access Journal. All articles are distributed under the terms of the Creative Commons Attribution NonCommercial License (http://creativecommons.org/licenses/by-nc/4.0/) which permits unrestricted non-commercial use, distribution, and reproduction in any medium, provided the original work is properly cited. 
ECAR [4], and IMPROVE trials [5], and from these studies, the 30-day mortality rates for EVAR were not significantly lower than open surgical repair. A meta-analysis of these data from individual-patient pooled data with extrapolation of survival outcomes to 90 days did not show any further benefit of EVAR over open repair [6]. However the 3-year outcomes of the IMPROVE trial showed a survival benefit in the mid-term for EVAR compared to open repair. It also showed an improvement in quality of life and cost-effectiveness of EVAR compared to open repair, and reintervention rates were not higher for EVAR, advocating its use for r-AAA [7]. These findings are significant since previous studies have mainly focused on the early perioperative outcomes of r-AAA, but once the patient is safely discharged from the hospital, the midterm outcomes favor EVAR over open repair.

The paradigm shift from open repair to EVAR for r-AAA repair is an ongoing process and despite the controversy in the literature, many major centers have implemented an EVAR first strategy for r-AAA, with open repair being performed only for patients with unsuitable anatomy for EVAR [8]. Based on these practice patterns, the most recent Society for Vascular Surgery practice guidelines on the care of patients with AAA have recommended EVAR over open repair for treatment of r-AAA if anatomically feasible, with a strong level of recommendation despite a low quality of evidence. Unfortunately not all centers have the necessary resources, dedicated staff and expertise to perform EVAR for r-AAA, factors which have been correlated with overall outcomes [9]. This difference in resource availability can exist among different centers, but also among different nations depending on the situation of each country in terms of medical resources, device availability

Table 1. Clinical and radiological characteristics of patients

\begin{tabular}{lc}
\hline \multicolumn{1}{c}{ Clinical finding } & Value \\
\hline Age $(y r)$ & $74.2(51-86)$ \\
Male sex & $10(76.9)$ \\
Symptoms & $11(84.7)$ \\
$\quad$ Abdominal pain & $1(7.7)$ \\
Loss of consciousness & $1(7.7)$ \\
Asymptomatic & $3(23.1)$ \\
Low blood pressure & $2(15.4)$ \\
Cardiopulmonary resuscitation & $2(15.4)$ \\
Hardman index ( $\geq 3)$ & $1(7.7)$ \\
Characteristics of AAA & $12(92.3)$ \\
Juxtarenal & $74.2(47.5-115.0)$ \\
Infrarenal & $102.8(55.0-135.0)$ \\
Maximal AAA diameter (mm) & $32.8(1.5-84.0)$ \\
AAA length (mm) & \\
Aortic neck length (mm) &
\end{tabular}

Values are presented as mean (range) or number (\%).

AAA, abdominal aortic aneurysm. or geographic location. This study aims to report the early experiences of consecutive EVAR cases performed for r-AAA in 2 tertiary centers in Korea and discuss the ways to implement a standardized protocol in the less than optimal Korean situation.

\section{METHODS}

Under the approval of the Institutional Review Board (IRB) (approval number: 21703693), patients who had undergone EVAR for r-AAA between May 2013 and December 2017 at 2 institutions (Seoul National University Bundang Hospital and The Catholic University of Korea Seoul St. Mary's Hospital) were retrospectively reviewed. Written informed consent was waived by the IRB. Preoperative data, such as demographics, initial symptoms, vital signs at presentation, and resuscitative method were collected. Preoperative images were reviewed for analysis of aneurysm morphology and characteristics, including aneurysm diameter, neck diameter, neck length, angulation, iliac artery involvement and other parameters related with suitability for EVAR. Intraoperative details included method and site of vessel access, use of aortic occlusion balloon, type of stent graft used, presence of endoleak on completion angiography, total procedural time, estimated blood loss, and amount of transfusion during the operation. Postoperative complications were also collected, such as cardiovascular events, pulmonary insufficiency, renal insufficiency, abdominal compartment syndrome, and 30-day mortality.

Ruptured AAA can show variability in clinical presentation. In this study we included all cases of free rupture with presence of hematoma in the retroperitoneal or intraabdominal space, as well as cases of symptomatic contained rupture or impending rupture. These were all identified on preoperative

Table 2. Operative and postoperative parameters

\begin{tabular}{lc}
\hline \multicolumn{1}{c}{ Parameter } & Value \\
\hline Operation time (min) & $140.46(72-255)$ \\
Transfusion of RBC (packs) & $2.8(0-14)$ \\
Devices & 12 \\
Bifurcated (Zenith, Cook) & 1 \\
AUI (Endurant, Medtronic) & 3 \\
Occlusion balloon use & 2 \\
Endoleak on completion angiography & 22.5 \\
Mean length of hospital stay (day) & \\
Mortality & 1 \\
AAA related & $1^{\text {a) }}$ \\
Non-AAA related & \\
\hline
\end{tabular}

Values are presented as mean (range) or number unless otherwise indicated.

RBC, red blood cells; AUI, aorto-uniiliac; AAA, abdominal aortic aneurysm.

${ }^{\text {a) }}$ Death by intracranial hemorrhage. 
CT images, and the decision to perform EVAR was left to the discretion of the attending surgeon. The protocol for management of these patients was similar to other reported studies, mainly involving liberal use of permissive hypotension if the patient's vital signs were unstable at presentation, and routine preoperative $\mathrm{CT}$ imaging if the patient had not had one from the referring hospital and if the vital signs allowed for it. As tertiary referral hospitals, most of the patients were referred from other hospitals after $\mathrm{CT}$ diagnosis, and in such cases the $\mathrm{CT}$ images were asked to be sent beforehand for early initiation of planning before patient arrival. This was a key factor since none of the hospitals had devices stocked in their shelves and therefore had to be brought to the hospital from other locations, which usually took a time interval of 1-1.5 hours. Once the patient was sent to the operation room, occlusion balloons were placed in selective cases and the procedure was performed either under general or local anesthesia. The rest of the procedure was performed in a similar fashion to routine elective EVAR, and bifurcated stent grafts were used for most of the procedures. Endoleaks present during completion angiography were corrected at the time of intervention, except for type II endoleaks, which were observed. After the operation, a laparotomy was liberally performed if there was any suspicion of abdominal compartment syndrome (ACS). ACS was diagnosed based on a clinical evaluation of the patients in the presence of organ dysfunction and any possibility of ongoing bleeding despite adequate stent graft deployment. Although the current guidelines define ACS as a sustained intraabdominal pressure (IAP) $>20 \mathrm{mmHg}$ (with or without an abdominal perfusion pressure $<60 \mathrm{mmHg}$ ) for repeated measurements [10], we did not routinely monitor bladder pressure to determine the IAP.

Table 3. Physician-modified EVAR in ruptured AAA

\begin{tabular}{cccc}
\hline No. Adjuvant procedures & $\begin{array}{c}\text { Distance from } \\
\text { aorta to renal } \\
\text { artery }(\mathrm{mm}), \\
\text { right : left }\end{array}$ & $\begin{array}{c}\text { Completion } \\
\text { angiography }\end{array}$ \\
\hline $1 \quad \begin{array}{c}\text { Right renal artery } \\
\text { fenestration, left renal } \\
\text { artery chimney } \\
\text { Left renal artery } \\
\text { fenestration }\end{array}$ & $9 / 11$ & $\begin{array}{c}\text { Type la } \\
\text { endoleak }\end{array}$ \\
\hline $2 \quad 15 / 0$ & $\begin{array}{c}\text { No } \\
\text { endoleak }\end{array}$ \\
\hline
\end{tabular}

EVAR, endovascular aneurysm repair; AAA, abdominal aortic aneurysm.
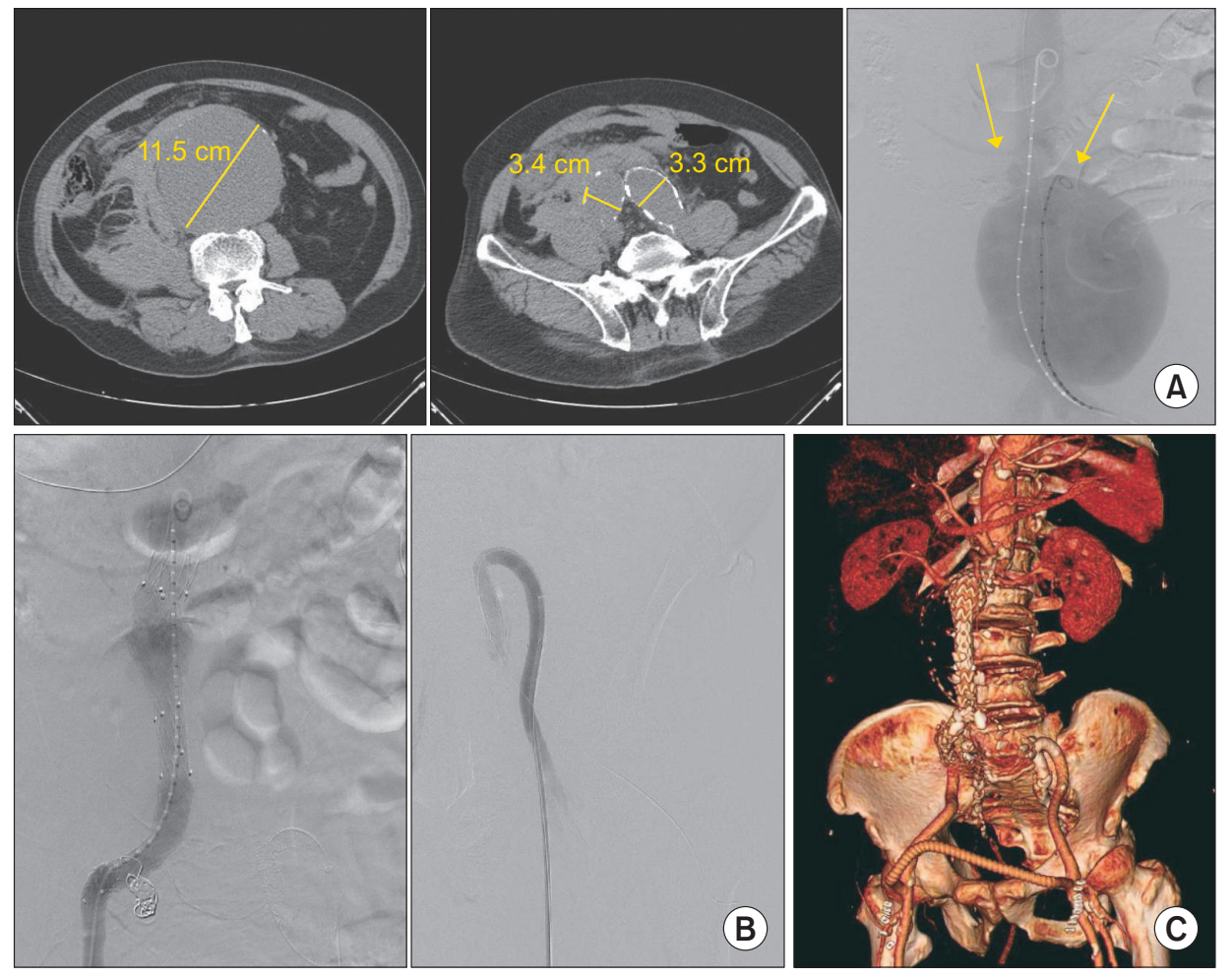

Fig. 1. (A) A case of ruptured $11.5 \mathrm{~cm}$ abdominal aortic aneurysm with concomitant bilateral common iliac and right internal iliac artery aneurysms. Initial angiography demonstrates a huge aneurysm with a relatively short aortic neck as shown by the location of both renal arteries (arrows). (B) The patient was treated with an aortouni-iliac device and crossover femorofemoral bypass, and an additional covered stent was inserted from the left external iliac artery into the left internal artery in a reversed U-shape configuration to allow for retrograde pelvic flow from the left femoral artery. (C) Follow-up CT reconstruction demonstrated a patent endograft with flow through the femoro-femoral graft into both the left internal iliac artery and the left lower extremity arterial system. 

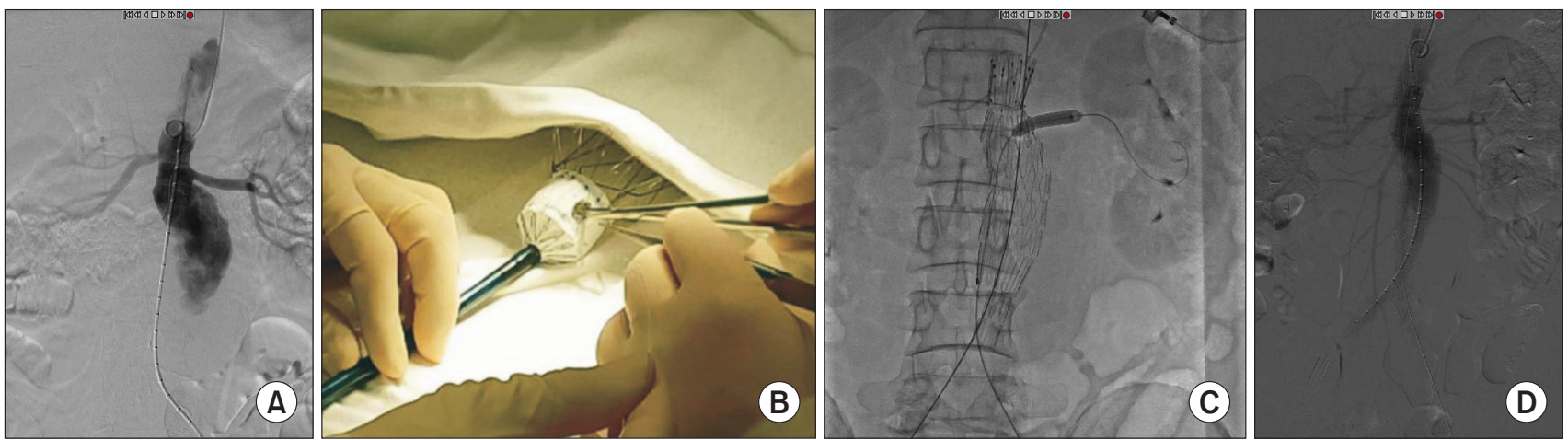

Fig. 2. (A) A case of advanced endovascular aneurysm repair for a ruptured 7.3-cm juxtarenal abdominal aortic aneurysm where the right renal artery was $15 \mathrm{~mm}$ below the left renal artery, as shown in the initial angiogram. (B) A single right renal fenestration was created in a bifurcated endograft, which was reinforced with a gold marker. (C) A self-expanding covered stent was deployed into the left renal artery after cannulation through the fenestration and post-dilated with a balloon. (D) Final angiogram showed good flow through both renal arteries with no evidence of endoleak.
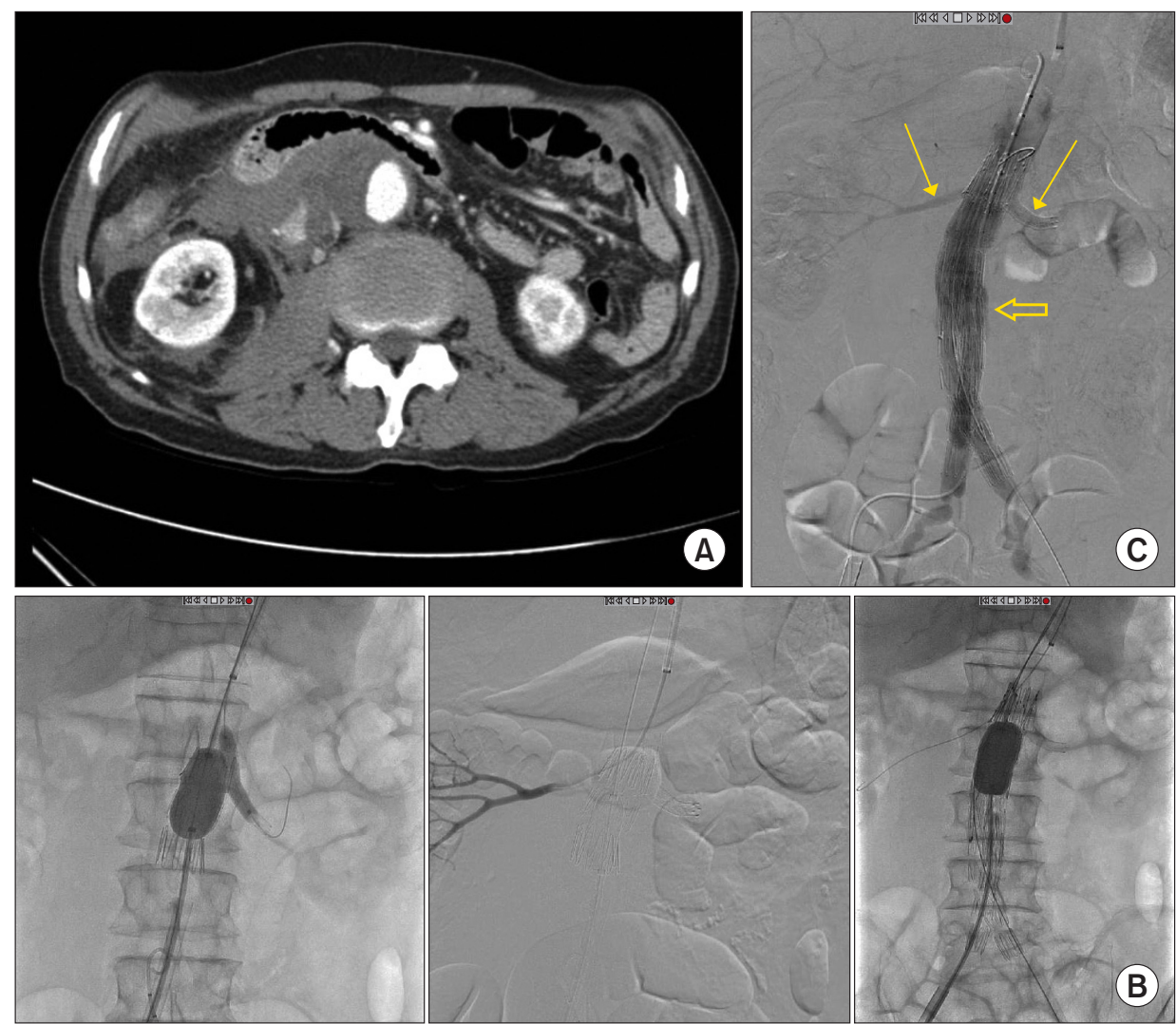

Fig. 3. (A) A case of advanced endovascular aneurysm repair for a ruptured 4.9-cm infrarenal abdominal aortic aneurysm with short neck (neck distance $9.4 \mathrm{~mm}$ from the right renal artery and $11.2 \mathrm{~mm}$ from the left renal artery). (B) A single renal fenestration was created on an extender endograft for the right renal artery and a chimney technique was performed for the left renal artery. The left chimney technique was performed successfully and the right renal artery was also successfully cannulated throught the endograft from a left brachial approach. However, after deployment of a bifurcated endograft with suprarenal fixation system, further insertion of a covered stent into the right renal artery was unsuccessful. (C) Despite failed cannulation through the fenestration into the right renal artery, flow through both renal arteries was preserved after endograft deployment (small arrows). Final angiogram showed a minor type la endoleak (or possibly III) which was observed (large arrow). Eventually the patient developed abdominal compartment syndrome and underwent explorative laparotomy with stent graft explantation and definitive surgery. 


\section{RESULTS}

From May 2013 to December 2017, 13 patients underwent emergent EVAR for r-AAA, comprising around $60 \%$ of total r-AAA repairs during the study period. The mean age of the patients was 74.2 years (range, 51-86). Eleven of the patients presented with abdominal or flank pain and 1 patient with loss of consciousness. Two patients underwent cardiopulmonary resuscitation at the Emergency Department (ER), and were immediately transferred to the operation room after successful resuscitation. One additional patient presented with unstable vital signs (systolic blood pressure $<60 \mathrm{mmHg}$ ), while the remaining patients showed relatively stable vital signs. The demographic data and the anatomical characteristics of the aneurysms are shown in Table 1.

General anesthesia was used in 12 patients, mean operative time was 140.5 minutes, and a mean 2.8 packs of red blood cell transfusion were needed per procedure (Table 2). An aortic occlusion balloon was used in 3 cases. There were 12 infrarenal and 1 juxtarenal $\mathrm{r}$-AAAs. All endografts used during the EVAR procedure were bifurcated Zenith Flex devices (Cook Medical Inc., Bloomington, IN, USA), except for 1 case where an aorto-uniiliac device (AUI) (Medtronic, Minneapolis, MN, USA) was used. The AUI with crossover femoro-femoral bypass was performed in a patient who underwent cardiopulmonary resuscitation at the ER and presented with a fusiform $11.5-\mathrm{cm}$ infrarenal AAA with bilateral common iliac and right internal iliac artery aneurysms (Fig. 1). An AUI was selected in order to save time from contralateral gate cannulation and thus control the bleeding as soon as possible. The device was extended to the right external iliac artery after right internal iliac artery embolization, and a crossover femoro-femoral bypass with prosthetic graft was performed. However, in order to preserve at least one iliac artery, a covered stent was inserted across the left internal and external arteries in a reversed U-shaped configuration from a left femoral approach, allowing for retrograde flow to the pelvis from the femoro-femoral bypass.

Two patients underwent advanced EVAR using physicianmodified endografts (Table 3). One of them involved a juxtarenal r-AAA patient that showed no neck between the aneurysm sac and the lowest left renal artery, but there was a difference of $15 \mathrm{~mm}$ between the right and left renal arteries (Fig. 2). Therefore, a single renal fenestration was made on the endograft and a covered stent (Viabahn $7 \times 29 \mathrm{~mm}$, WL Gore \& Associates, Flagstaff, AZ, USA) was inserted through the fenestration into the left renal artery. Another patient presented with a short neck infrarenal r-AAA, with the aneurysm being $9.4 \mathrm{~mm}$ away from the right renal artery and $11.2 \mathrm{~mm}$ from the left renal artery (Fig. 3). Therefore a fenestration was created for the right renal artery and a chimney technique was used for the left renal artery. Unfortunately, cannulation of the right renal artery through the fenestration was unsuccessful, and therefore a covered stent could not be inserted, yet the right renal artery flow was well preserved and there was good

Table 4. Postoperative complications and management $(<30$ days)

\begin{tabular}{|c|c|c|}
\hline Variable & $\begin{array}{c}\text { Successful } \\
\text { EVAR } \\
(\mathrm{n}=13)\end{array}$ & Management \\
\hline $\mathrm{AMI} /$ stroke & 1 & $\begin{array}{l}\text { ROSC after cardiac } \\
\text { compression }\end{array}$ \\
\hline Pneumonia & 1 & Conservative management \\
\hline Acute kidney injury & 1 & Conservative management \\
\hline Bowel ischemia & 1 & Hartmann operation \\
\hline $\begin{array}{l}\text { Abdominal } \\
\text { compartment } \\
\text { syndrome }\end{array}$ & 3 & $\begin{array}{l}2 \text { Decompressive laparotomy } \\
1 \text { Refused surgical treatment }\end{array}$ \\
\hline
\end{tabular}

EVAR, endovascular aneurysm repair; AMI, acute myocardial infarction; ROSC, return of spontaneous circulation.
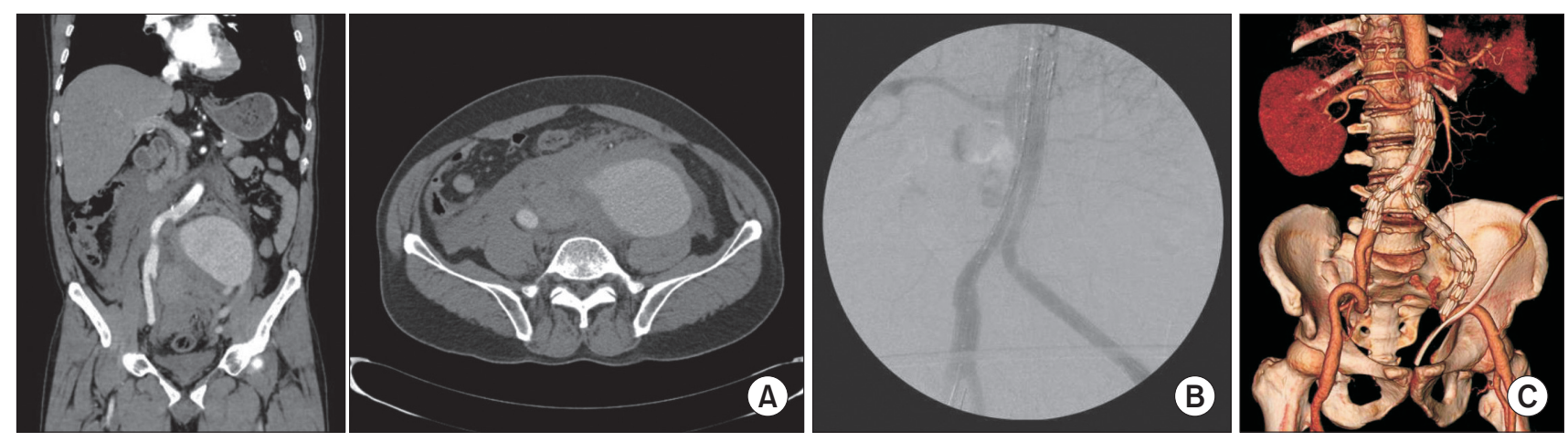

Fig. 4. (A) A case of a ruptured 10.7-cm left common iliac artery aneurysm treated by endovascular aneurysm repair (EVAR). (B) An EVAR procedure was performed with extension of the left limb graft to the external iliac artery. Final angiogram shows good exclusion of the aneurysm without evidence of endoleak. The patient developed abdominal compartment syndrome and therefore underwent emergent decompressive laparotomy with retroperitoneal hematoma evacuation and surgical ligation of the left internal artery. (C) Follow-up CT on postoperative day 14 demonstrates a patent graft without any evidence of endoleak. 
apposition between the proximal part of the stent graft and the aortic wall. On final angiography, there was a small amount of type Ia (or possibly III) endoleak present, even after repeated ballooning with a molding balloon. We decided to observe this finding as the amount of endoleak was small. However the patient developed a drop in blood pressure the day after the operation, and was diagnosed with ACS due to suspected ongoing bleeding. Hence, the patient underwent decompressive laparotomy with stent graft explantation and corrective surgery.

In our study, successful deployment of an endovascular stent graft during the primary procedure was achieved in $100 \%$ (13 of 13). The overall 30-day mortality was $7.7 \%$ (1 of 13), and the mean length of hospital stay was 22.5 days. Early postoperative complications are shown in Table 4, which included 3 cases of ACS. All ACS occurred within 48 hours of the initial procedure, showing signs of hypotension, decreased hemoglobin and organ dysfunction. Two of these patients underwent emergency decompressive laparotomy, which showed massive retroperitoneal hematoma, while 1 patient refused any kind of invasive treatment and eventually died from multiorgan failure on postoperative day 2 (the only mortality in our case series). One of the patients with ACS who underwent decompressive laparotomy was the case of physician-modified endograft for the AAA with short aortic neck described previously, while the other patient initially presented with a $10.7 \mathrm{~cm}$ left common iliac artery aneurysm on angiography (Fig. 4). In this patient, an aortic occlusion balloon was used due to unstable vital signs, and despite the hostile location and size of the aneurysm, we successfully deployed a bifurcated stent graft by endovascular means without any evidence of endoleak on completion angiography. However, the patient presented with clinical signs of ACS, and therefore a decompressive laparotomy was performed on the same day with ligation of the left internal iliac artery and evacuation of the retroperitoneal hematoma.

\section{DISCUSSION}

With technical advancements in EVAR technology, mortality rates after treatment of r-AAA with EVAR have declined as well as the length of hospitalization [6,11,12]. Based on the current trend of favoring EVAR for r-AAA, both our centers adopted an EVAR-first strategy for r-AAA when indicated. The indication for selecting EVAR was at the discretion of the attending surgeon, which included factors such as patient conditions, anatomic suitability and device availability, among others. Despite the unavailability of stocked EVAR devices in our centers, our favorable geographic locations allowed for delivery of the devices within a maximum of 2 hours. A door-to-intervention time of less than 90 minutes has been proposed as a goal for successful treatment of r-AAA, and for cases of referral from other regional centers we implemented a protocol of obtaining and reviewing the $\mathrm{CT}$ images before arrival of the patient. In this way, the necessary devices were ordered early enough so that they could be implanted on time. Based on this protocol, we achieved a $100 \%$ technical success in our 13 cases of EVAR. Two of these cases included advanced EVAR techniques for anatomically unsuitable cases with short aneurysmal neck, where physician-modified fenestration and chimney techniques were applied. These advanced techniques cannot be performed for all r-AAA cases and should only be used selectively based on patient conditions. Additionally it requires a great amount of experience in planning and execution, which is usually obtained from elective cases.

One of the major complications after EVAR for r-AAA which requires a high index of suspicion is ACS. Although many studies have been performed on EVAR for r-AAA, there is still little information about the incidence of acute ACS after EVAR for r-AAA. In our study, there were 3 patients (23\%) diagnosed of ACS, which was slightly higher than previously reported range of 5.5\%-21\% [13-15]. Two of these patients underwent decompressive laparotomy immediately after the detection of ACS, while one patient refused further surgery. We did not routinely monitor intraabdominal (bladder) pressure, but the use of bladder pressure does not always accurately reflect a state of ACS, and ACS can be diagnosed by clinical presentation alone in the presence of multiorgan failure, hypotension and drop in hemoglobin levels. Treatment for ACS after EVAR is still under controversy, and although medical management may be attempted first, surgical management should be performed without delay if there is no improvement in clinical presentation [16]. Our threshold for decompressive laparotomy was low and by performing decompression at an early stage, our ACS patients recovered well without any other acute complications.

The presence of endoleak after EVAR for r-AAA should also be noted, since it is associated with worse outcomes than in non-ruptured AAA [17,18]. In our case series, there was 1 case of endoleak (type Ia) on completion angiography, which was thought to be minor during the procedure, but eventually required decompressive laparotomy due to progression of ACS from probable ongoing bleeding. There was one other case in which a type I endoleak was suspected after stent graft implantation and an extender stent graft was additionally inserted, but a persistent endoleak (type III or IV) was still noted, and therefore an additional stent graft was relined to solve the problem. This case showed no endoleak during the follow-up and the patient recovered without complications. These examples show how endoleaks during r-AAA can be more devastating and can undergo rapid progression, and therefore should be dealt with much more aggressively during the initial procedure, either by endovascular means or open surgical repair (with the exception of minor type II endoleaks). 
This study shows the early experiences of EVAR for r-AAA in two major centers in Korea. Although the numbers are small, it shows the outcomes of consecutive EVAR cases for r-AAA, and the results are fairly promising. These results may be prone to selection bias, since it does not include all cases of r-AAA treatment during the study period, and a comparative analysis against cases of open r-AAA repair with larger numbers may be needed. EVAR may have been attempted more frequently for concealed or impending rupture cases, which may present with relatively more stable vital signs on initial presentation. Despite these limitations, most of the previous studies of EVAR for r-AAA were performed in large volume centers with high incidence of AAA. Although the benefit of EVAR for r-AAA is still debatable, most experts agree that good outcomes for EVAR can only be achieved in large volume centers with the necessary experience, technical support and dedicated staff trained for r-AAA treatment [19-21]. However, in places where the incidence of AAA is lower, such as in Korea, it is more difficult to have a dedicated staff and stocked devices, and experience to treat r-AAA by EVAR may be lacking, even in major centers. A recent study analyzing multiple international vascular registries demonstrated that perioperative mortality rates were lower in higher volume centers compared to lower volume centers, but this difference was mainly attributed to a difference in open surgical repair, while there was no significant difference in perioperative mortality for EVAR across different volume centers [22]. Our study is in accordance in that we have demonstrated the feasibility of EVAR for r-AAA in Korea where the incidence of AAA is relatively lower, as long as an experienced and well-trained staff is available. The technical support is also very important, with a good hybrid operation room and the necessary devices readily available on time for execution of the procedure. Although we did not have stocked stent graft devices in our centers, availability of stocked devices seems very important if EVAR is to be attempted more routinely and to achieve more optimal outcomes. The use of advanced EVAR techniques such as sandwich techniques and/or physician- modified endografts with fenestrations and scallops can also provide satisfactory results in emergent situations especially if the patient is high-risked and unfit for open repair. Although these techniques may be outside the instructions-for-use of the devices, they can become very handy when treating these high risk and hemodynamically unstable patients as a means of rapid bleeding control without compromising visceral or renal perfusion and avoiding open repair. The long-term durability of physician-modified endografts is still to be determined but in the acute setting, it may be a good option as a life-saving procedure to achieve effective hemostasis and hemodynamic stability. Another limitation of our study is that most of our cases were performed under general anesthesia, which may offset the advantage of EVAR being able to be performed under local anesthesia. Although local anesthesia is generally favored for EVAR during r-AAA treatment, the choice of anesthesia may vary according to the situation of each individual center. As more experience is obtained with EVAR for r-AAA, more liberal use of local anesthesia with backup support from the anesthesiologists may be implemented as protocol.

In conclusion, the present study reported a 30-day mortality of $7.7 \%$ for a total of 13 cases of EVAR for r-AAA. The results support previous studies that show EVAR is a feasible treatment option in r-AAA patients. EVAR can also be performed for r-AAA with hostile anatomies by use of advanced techniques in selected cases. Moreover, our results demonstrate the importance of early intervention, such as decompressive laparotomy for suspected ACS or aggressive management of endoleaks intraoperatively. Finally the continuous efforts to maintain a dedicated team of experts and technical support are critical in achieving good results.

\section{CONFLICTS OF INTEREST}

No potential conflict of interest relevant to this article was reported.

\section{REFERENCES}

1. Aggarwal S, Qamar A, Sharma V, Sharma A. Abdominal aortic aneurysm: a comprehensive review. Exp Clin Cardiol 2011; 16:11-5.

2. Harris LM, Faggioli GL, Fiedler R, Curl GR, Ricotta JJ. Ruptured abdominal aortic aneurysms: factors affecting mortality rates. J Vasc Surg 1991;14:812-8.
3. Reimerink JJ, Hoornweg LL, Vahl AC, Wisselink W, van den Broek TA, Legemate DA, et al. Endovascular repair versus open repair of ruptured abdominal aortic aneurysms: a multicenter randomized controlled trial. Ann Surg 2013;258:24856.

4. Desgranges $\mathrm{P}$, Kobeiter H, Katsahian
S, Bouffi M, Gouny P, Favre JP, et al. Editor's Choice - ECAR (Endovasculaire ou Chirurgie dans les Anevrysmes aortoiliaques Rompus): a french randomized controlled trial of endovascular versus open surgical repair of ruptured aortoiliac aneurysms. Eur J Vasc Endovasc Surg 2015:50:303-10. 
5. IMPROVE Trial Investigators, Powell JT, Sweeting MJ, Thompson MM, Ashleigh $\mathrm{R}$, Bell R, et al. Endovascular or open repair strategy for ruptured abdominal aortic aneurysm: 30 day outcomes from IMPROVE randomised trial. BMJ 2014; 348:f7661.

6. Sweeting MJ, Balm R, Desgranges P, Ulug P, Powell JT; Ruptured Aneurysm Trialists. Individual-patient meta-analysis of three randomized trials comparing endovascular versus open repair for ruptured abdominal aortic aneurysm. $\mathrm{Br}$ Surg 2015;102:1229-39.

7. IMPROVE Trial Investigators. Comparative clinical effectiveness and cost effectiveness of endovascular strategy $\mathrm{v}$ open repair for ruptured abdominal aortic aneurysm: three year results of the IMPROVE randomised trial. BMJ 2017; 359:j4859.

8. Mureebe L, Egorova N, Giacovelli JK, Gelijns A, Kent KC, McKinsey JF. National trends in the repair of ruptured abdominal aortic aneurysms. J Vasc Surg 2008:48:1101-7.

9. Lesperance K, Andersen C, Singh N, Starnes B, Martin MJ. Expanding use of emergency endovascular repair for ruptured abdominal aortic aneurysms: disparities in outcomes from a nationwide perspective. J Vasc Surg 2008;47:1165-70; discussion 1170-1.

10. Kirkpatrick AW, Roberts DJ, De Waele J, Jaeschke R, Malbrain ML, De Keulenaer $B$, et al. Intra-abdominal hypertension and the abdominal compartment syndrome: updated consensus definitions and clinical practice guidelines from the World Society of the Abdominal Compartment Syndrome. Intensive Care Med 2013;39:1190-206.

11. Badger SA, Harkin DW, Blair PH, Ellis PK, Kee F, Forster R. Endovascular repair or open repair for ruptured abdominal aortic aneurysm: a Cochrane systematic review. BMJ Open 2016;6:e008391.

12. IMPROVE Trial Investigators. Endovascular strategy or open repair for ruptured abdominal aortic aneurysm: one-year outcomes from the IMPROVE randomized trial. Eur Heart J 2015;36:2061-9.

13. Karkos CD, Menexes GC, Patelis N, Kalogirou TE, Giagtzidis IT, Harkin DW. A systematic review and meta-analysis of abdominal compartment syndrome after endovascular repair of ruptured abdominal aortic aneurysms. J Vasc Surg 2014:59:829-42.

14. Acosta S, Lindblad B, Zdanowski Z. Predictors for outcome after open and endovascular repair of ruptured abdominal aortic aneurysms. Eur J Vasc Endovasc Surg 2007;33:277-84.

15. Rubenstein C, Bietz G, Davenport DL, Winkler M, Endean ED. Abdominal compartment syndrome associated with endovascular and open repair of ruptured abdominal aortic aneurysms. J Vasc Surg 2015;61:648-54.

16. Chen X, Zhao J, Huang B, Yuan D, Yang Y, Ma Y. Abdominal compartment syndrome after endovascular repair for ruptured abdominal aortic aneurysm leads to acute intestinal necrosis: Case report. Medicine (Baltimore) 2016;95:e5316.
17. Powell JT, Sweeting MJ, Ulug P, Thompson MM, Hinchliffe RJ; IMPROVE Trial Investigators. Editor's choice - re-interventions after repair of ruptured abdominal aortic aneurysm: a report from the IMPROVE randomised trial. Eur J Vasc Endovasc Surg 2018:55:625-32.

18. Quinn AA, Mehta M, Teymouri MJ Keenan ME, Paty PSK, Zhou Y, et al. The incidence and fate of endoleaks vary between ruptured and elective endovascular abdominal aortic aneurysm repair. J Vasc Surg 2017;65:1617-24.

19. Wong LA, Milner R. Interhospital Transfer of Ruptured AAA's for EVAR: preposterous or prudent? Vasc Endovascular Surg 2016; 50:455-7.

20. Mehta M, Taggert J, Darling RC 3rd, Chang BB, Kreienberg PB, Paty PS, et al. Establishing a protocol for endovascular treatment of ruptured abdominal aortic aneurysms: outcomes of a prospective analysis. J Vasc Surg 2006;44:1-8; discussion 8

21. Moore R, Nutley M, Cina CS, Motamedi M, Faris P, Abuznadah W. Improved survival after introduction of an emergency endovascular therapy protocol for ruptured abdominal aortic aneurysms. J Vasc Surg 2007:45:443-50.

22. Budtz-Lilly J, Bjorck M, Venermo M, Debus S, Behrendt CA, Altreuther M, et al. Editor's choice - the impact of centralisation and endovascular aneurysm repair on treatment of ruptured abdominal aortic aneurysms based on international registries. Eur J Vasc Endovasc Surg 2018; 56:181-8 